\title{
Shortened Coagulation Profile in Pregnancy
}

Dr. Balaji. ${ }^{1^{*}}$, Dr. Kalla Ravi Teja ${ }^{2}$, Dr. Govindaraj.T ${ }^{3}$, B. Amirtha Sivani ${ }^{4}$

\begin{abstract}
${ }^{1}$ Senior Resident, Department of Pathology, Sri Venkateshwaraa Medical College and Research Centre, Pondicherry, India ${ }^{2}$ Assistant Professor, Department of Pathology, Sri Venkateshwaraa Medical College and Research Centre, Pondicherry, India ${ }^{3}$ Professor and HOD Department of Pathology, Sri Venkateshwaraa Medical College and Research Centre, Pondicherry, India ${ }^{4}$ Final Year MBBS, Sri Venkateshwaraa Medical College and Research Centre, Pondicherry, India
\end{abstract}

DOI: $10.36347 /$ sjams.2022.v10i01.018

| Received: 07.12.2021 | Accepted: 15.01.2022 | Published: 24.01.2022

*Corresponding author: Dr. Balaji. S

Senior Resident, Department of Pathology, Sri Venkateshwaraa Medical College and Research Centre, Pondicherry India

\section{Abstract}

\section{Original Research Article}

Introduction: Hyper-coagulation and low grade disseminated intravascular coagulation exists during the entire period of pregnancy and immediate postpartum period. The hormonal changes in pregnancy maintains the placental function during the entire pregnancy and also during delivery, this is achieved by the significant changes in coagulation system during pregnancy; increases in levels of clotting factors, decreased concentration of anticoagulants and diminishing fibrinolytic activities, leading to the state of hypercoagulability. Study and result: The study was done in Central lab, Department of Pathology of our Institute. In this study, the PT and APTT levels of 150 pregnant women during different trimesters of pregnancy and labor were studied and we got a outcome of shortened profile in 104 patients out of which cases with shortened PT is $34 \%$, shortened APTT is $51 \%$, shortened PT and APTT is $15 \%$. Implication: With the knowledge of the reference level, Shortened APTT independently predicts the first episodes and also subsequent recurrence of venous thromboembolism (VTE) and Post-partum hemorrhage.

Keywords: Coagulation profile, pregnancy.

Copyright $(\odot) 2022$ The Author(s): This is an open-access article distributed under the terms of the Creative Commons Attribution 4.0 International License (CC BY-NC 4.0) which permits unrestricted use, distribution, and reproduction in any medium for non-commercial use provided the original author and source are credited.

\section{INTRODUCTION}

Hyper-coagulation and low grade disseminated intravascular coagulation exists during the entire period of pregnancy and immediate postpartum period [1]. The hormonal changes in pregnancy maintains the placental function during the entire pregnancy and also during delivery, this is achieved by the significant changes in coagulation system during pregnancy; increases in levels of clotting factors, decreased concentration of anticoagulants and diminishing fibrinolytic activities, leading to the state of hypercoagulability [2].

Concentrations of coagulation factors V, VII, VIII, IX, X, XII and von Willebrand factor are significantly increased in pregnancy along with increased concentration of plasma fibrinogen 2 . These changes in coagulation and fibrinolysis are attributed to estradiol induced alterations of triglycerides [3]. Prothrombin time and activated partial thromboplastin time respectively measures the extrinsic and intrinsic pathway of coagulation [4].

Activated partial thromboplastin time is used to assess the coagulation disorders in patient with abnormal bleeding due to deficiencies in clotting factors, monitoring anticoagulant therapy and detecting inhibitors of blood coagulation [5].

Recent studies suggest, a shortened activated partial thromboplastin time might actually reflect invivo events that might be associated with hypercoagulability. Also shortened activated partial thromboplastin time has long been thought as an artifact secondary to problematic blood collection, storage or processing leading to in-vitro activation [6].

Previous studies done have documented a shortened activated partial thromboplastin time levels during pregnancy [7, 8], this can be explained by increase in levels of F V, VIII, IX and XII [8]. Authors have also formulated normative value of coagulation parameters according to the period of gestation [9-11].

\section{AIM}

To find the prevalence of shortened prothrombin time (PT) and activated partial thromboplastin time (APTT) in pregnant women. 


\section{Methodology}

Study design: Cross sectional Study.

Study Setting: Sri Venkateshwaraa medical college hospital and research Centre, Puducherry.

\section{Study population}

Antenatal mothers attending the OPD, Department of Obstetrics and Gynecology

\section{Inclusion criteria}

Pregnant women at any gestational age

\section{Exclusion criteria}

- Women with active bleeding

- Antenatal haemorrhage during present/past pregnancy

- Personal/ family history of known bleeding diathesis

- History of taking anticoagulants

Sample size: Period Sample

All the women attending the outpatient department during the study Period (2 months) fulfilling the eligibility criteria will be included.
Procedure: The study was done in Central lab, Department of Pathology of our Institute. All pregnant mothers attending the OPD in Obstetrics department were explained about the study and written informed consent was obtained. Clinical details of the pregnant women was taken and categorized as group I, II and III based on their trimester.

Blood Sample Collection: Under aseptic precautions, $2 \mathrm{ml}$ of venous blood was drawn from the ante-cubital vein and stored in Sodium citrate $(3.2 \%)$ vacutainer, with proper labelling and analyzed within thirty minutes of collection.

Sample Analysis: The collected samples were centrifuged for 15 minutes at $2000 \mathrm{rpm}$ and the plasma was collected and analyzed with ERBA ECL 105, Semiautomated coagulometer. PT and APTT values were obtained.

Quality Control: The Coagulometer is under external quality control program of CMC, Vellore. The internal quality check is done routinely by running the quality control samples with each batch of test samples.

STATISTICAL ANALYSIS: $\mathbf{N}=\mathbf{1 5 0}$

Duration: 2 months

\begin{tabular}{|l|l|l|}
\hline PT/APTT & NUMBER & PERCENTAGE \% \\
\hline NORMAL OR INCREASED & 46 & 31 \\
\hline SHORTENED PT & 34 & 23 \\
\hline SHORTENED APTT & 55 & 36 \\
\hline BOTH SHORTENED & 15 & 10 \\
\hline
\end{tabular}

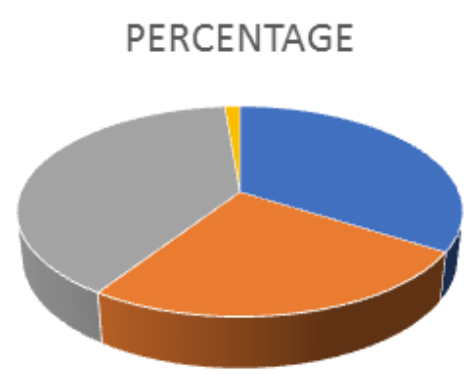

- NORMAL OR INCREASED PT $=$ SHORTENED PT

॥ SHORTENED APTT = $\quad$ SHORTENED PT AND APTT

\begin{tabular}{|l|l|l|l|l|l|}
\hline $\begin{array}{l}\text { S. } \\
\text { NO }\end{array}$ & $\begin{array}{l}\text { PREGNANCY } \\
\text { STAGES }\end{array}$ & $\begin{array}{l}\text { SHORTENED } \\
\text { PT }\end{array}$ & $\begin{array}{l}\text { SHORTENED } \\
\text { APTT }\end{array}$ & $\begin{array}{l}\text { BOTH PT AND APTT } \\
\text { SHORTENED }\end{array}$ & TOTAL \\
\hline 1. & $1-2$ TRIMESTER & 4 & 5 & 3 & 12 \\
\hline 2. & $3^{\text {RD }}$ TRIMESTER & 25 & 46 & 10 & 81 \\
\hline 3. & LABOUR & 5 & 4 & 2 & 11 \\
\hline 4. & TOTAL & 34 & 55 & 15 & 104 \\
\hline
\end{tabular}




\section{RESULT}

In this study, the PT and APTT levels of 150 pregnant women during different trimesters of pregnancy and labor were studied and we got a outcome of shortened profile in 104 patients out of which cases with shortened PT is 34\%, shortened APTT is $51 \%$, shortened PT and APTT is $15 \%$.

\section{REVIEW OF LITERATURE}

- SarikaS et al. [1]. In New Delhi, 2016, conducted a study to assess the prevalence of shortened prothrombin time and activated partial thromboplastin time in pregnant women. The results of the study were Among 173 pregnant women, $71.7 \%$ were detected to have shortened PT, APTT or both. The commonest abnormality was an isolated shortened aPTT.

- Mina A et al. [12] conducted a study on laboratory evaluation into the short APTT (2010). The results of the study were on patients presenting with short APTT' in reflective of complex hyper coagulant milieu that could feasibly contribute to thrombotic risk.

- Ibeh $\mathrm{N}$ et al. [7] in 2015 conducted a study at Nigeria, to evaluate the influence of normal pregnancy on blood coagulation and to explore changes in activity from first through the third trimester. The result of the study was "The means of APTT were significantly lower in the first, second and third trimester compared with controls".

- Hammerova $\mathrm{H}$ et al. [8] in 2014 conducted a study to establish the physiologic changes in haemostasis during pregnancy among 148 pregnant women. The results of the study were PT, APTT and INR were significantly shorter.

\section{CONCLUSION}

The present study shows a very high prevalence of shortened PT and APTT during 3rd trimester of pregnancy and labor when chances of bleeding are highest. Further studies with larger cohorts and other coagulation parameters are required to predict clinical outcomes in pregnant women with shortened coagulation profile.

\section{IMPLICATIONS}

With the knowledge of the reference level, Shortened APTT independently predicts the first episodes and also subsequent recurrence of venous thromboembolism (VTE) and Post-partum hemorrhage. Thrombophilia predisposes women for an increased risk of developing both early and late complications in pregnancy, which includes pre-mature rupture of membranes (PROM), Pre-eclampsia, and intra uterine growth restrictions.

\section{REFERENCES}

1. Singh, S., Rishi, B., \& Sharma, S. (2016). Shortened coagulation profile in pregnancycomparative analysis in different trimestersretrospective study from a tertiary care centre. Eur J Pharm Med Res, 3, 548-51.

2. Bremme, K.A. (2003). Haemostatic changes in pregnancy, Best Pract Res ClinHaematol, 16(2); 153-68.

3. Sattar, N., Greer, I. A., Rumley, A., Stewart, G., Shepherd, J., Packard, C. J., \& Lowe, G. D. (1999). A longitudinal study of the relationships between haemostatic, lipid, and oestradiol changes during normal human pregnancy. Thrombosis and haemostasis, 81(01), 71-75.

4. Khan, H. A., Alhomida, A. S., Al Rammah, T. Y., Sobki, S. H., Ola, M. S., \& Khan, A. A. (2013). Alterations in prothrombin time and activated partial thromboplastin time in patients with acute myocardial infarction. International journal of clinical and experimental medicine, 6(4), 294.

5. Tripodi, A., \& Mannucci, P. M. (2006). Activated partial thromboplastin time (APTT). New indications for an old test?. Journal of Thrombosis and Haemostasis, 4(4), 750-751.

6. Ten Boekel, E., Böck, M., Vrielink, G. J., Liem, R., Hendriks, H., \& de Kieviet, W. (2007). Detection of shortened activated partial thromboplastin times: an evaluation of different commercial reagents. Thrombosis research, 121(3), 361-367.

7. Ibeh, N., Okocha, C. E., Aneke, C. J., Onah, C. E., Nwosu, A. O., \& Nkwazema, K. A. (2015). Normal pregnancy and coagulation profile: from the first through the third trimester. Nigerian journal of medicine: journal of the National Association of Resident Doctors of Nigeria, 24(1), 54-57.

8. Hammerova, L., Chabada, J., Drobny, J., \& Batorova, A. (2014). Longitudinal evaluation of markers of hemostasis in pregnancy. Bratislavske lekarske listy, 115(3), 140-144.

9. Liu, X. H., Jiang, Y. M., Shi, H., Yue, X. A., Wang, Y. F., \& Yang, H. (2009). Prospective, sequential, longitudinal study of coagulation changes during pregnancy in Chinese women. International Journal of Gynecology \& Obstetrics, 105(3), 240-243.

10. Szecsi, P. B., Jørgensen, M., Klajnbard, A., Andersen, M. R., Colov, N. P., \& Stender, S. (2010). Haemostatic reference intervals in pregnancy. Thrombosis and haemostasis, 103(04), 718-727.

11. Uchikova, E. H., \& Ledjev, I. I. (2005). Changes in haemostasis during normal pregnancy. European Journal of Obstetrics \& Gynecology and Reproductive Biology, 119(2), 185-188.

12. Mina, A., Favaloro, E. J., Mohammed, S., \& Koutts, J. (2010). A laboratory evaluation into the short activated partial thromboplastin time. Blood coagulation \& fibrinolysis, 21(2), 152-157. 